\title{
CONSIDERACIONES CONCEPTUALES PARA EL ESTUDIO SOCIOCULTURAL DE LAS ORGANIZACIONES JUVENILES PARTIDARIAS ${ }^{1}$
}

JUAN R. GRANDINETTI*

\begin{abstract}
RESUMEN
A diferencia de lo ocurrido en el resto de América Latina, en la última década, Argentina ha asistido a un proceso de revitalización de las organizaciones de militancia juvenil partidaria. Este fenómeno ha despertado el interés académico, dando lugar a la emergencia de un campo de estudios. El presente artículo busca realizar un aporte conceptual para la investigación empírica de esta modalidad de participación política juvenil. Luego de una revisión de la literatura sobre Argentina y otros países latinoamericanos, se presentarán una serie de propuestas para el estudio de este tipo de organizaciones, a partir del análisis de su medio social, y de las dimensiones cultural, organizacional y generacional de lo juvenil. Así, el artículo discute algunas herramientas conceptuales para indagar en los ámbitos de reclutamiento y sociabilidad de las organizaciones partidarias juveniles, en los usos y sentidos partidarios de la juventud, y en los procesos de movilización e involucramiento político de los militantes.
\end{abstract}

PALABRAS CLAVE: PARTICIPACIÓN POLÍTICA JUVENIL, PARTIDOS POLÍTICOS, ACTIVISMO

\section{CONSIDERAÇÕES CONCEITUAIS PARA O ESTUDO SOCIOCULTURAL DAS ORGANIZAÇÕES JUVENIS PARTIDÁRIAS}

\section{RESUMO}

Ao contrário do que aconteceu nos outros lugares da América Latina, na última década a Argentina presenciou um processo de revitalização das organizações de militância partidária juvenil. Este fenómeno despertou o interesse acadêmico, dando origem à emergência de um campo de estudos. O presente artigo visa realizar uma contribuição conceitual para a pesquisa empírica desta modalidade de participação política juvenil. Depois de uma revisão de literatura sobre a Argentina e outros países da América Latina, serão apresentadas uma série de propostas para o estudo deste tipo de organizações, a partir da análise de seu ambiente social, e das dimensões cultural, organizacional e geracional do juvenil. Assim, o artigo discute algumas ferramentas conceituais para pesquisar nas áreas de recrutamento e sociabilidade das organizações

\footnotetext{
${ }^{1}$ Este artículo sistematiza el abordaje teórico-metodológico y sintetiza el estado de la cuestión de una investigación doctoral sobre militancias juveniles partidarias en la Argentina reciente, desarrollada en la Universidad de Buenos Aires entre 2013 y 2018, y financiada a través de becas del Consejo Nacional de Investigaciones Científicas y Técnicas de Argentina (CONICET), la Universidad Nacional de Avellaneda, el Grupo Coimbra y el Gobierno de Canadá.

* Doctorando en Ciencias Sociales por la Universidad de Buenos Aires, Argentina. Magíster en Ciencia Política por la Universidad Nacional de San Martín, Argentina, y Licenciado y Profesor en Sociología por la Universidad de Buenos Aires. Docente de la Facultad de Ciencias Sociales de la Universidad de Buenos Aires. E-mail: jgrandinetti@sociales.uba.ar
} 
partidárias juvenis, nos usos e sentidos partidários da juventude, e nos processos de mobilização e engajamento político dos militantes.

PALAVRAS-CHAVE: PARTICIPAÇÃO POLÍTICA JUVENIL, PARTIDOS POLÍTICOS, ATIVISMO

\title{
CONCEPTUAL CONSIDERATIONS TO A SOCIOCULTURAL STUDY OF POLITICAL PARTY YOUTH ORGANIZATIONS
}

\begin{abstract}
In contrast to the events happened in other Latin-American countries, Argentina has experienced a revitalization of political party youth organizations in the last decade. This phenomenon has aroused academic attention, giving place to the emergence of a field of studies. The present article aims to be a conceptual contribution to the empirical research of this form of youth political participation. After a literature review about youth participation within political parties in Argentina and other Latin-American countries, the article presents conceptual suggestions for the study of these organizations, focusing on both their social environment and on the cultural, organizational and generational dimensions of youth. Hence, the article discusses some conceptual tools for exploring the recruitment and sociability spaces of political party youth organizations, in its partisan uses and meanings of youth, and on the engagement and mobilization processes of young activists.
\end{abstract}

KEYWORDS: YOUTH POLITICAL PARTICIPATION, POLITICAL PARTIES, ACTIVISM.

\section{INTRODUCCIÓN}

Mientras que los estudios sobre participación juvenil en América Latina y el resto del mundo se han ocupado de señalar la aparición de nuevas formas de involucramiento y participación política entre los jóvenes, con un consecuente distanciamiento de las prácticas tradicionales de la militancia partidaria, Argentina ha asistido, en la última década, a un proceso de revitalización de las organizaciones juveniles vinculadas a los partidos políticos. Así, no solo han surgido nuevos espacios juveniles partidarios, sino que estas militancias han ganado una gran visibilidad en el espacio público y en el discurso de la dirigencia política. A contramano de lo ocurrido en otros países, se ha constatado en Argentina una recuperación relativa de los niveles de confianza de la ciudadanía en los partidos políticos, si se los compara con los de la década de los noventas y los primeros años de los dos mil. Esta recuperación tuvo un mayor impacto entre las generaciones jóvenes, ${ }^{2}$ que fueron exitosamente interpeladas y movilizadas

\footnotetext{
${ }^{2}$ Un estudio realizado por WVS muestra que los encuestados que confiaban mucho o bastante en los partidos políticos eran el 8,2\% en 1995; 7\% en 1999; 7,6\% en 2006 y 15\% en 2013. En este último año, el grupo etario de los menores de 30 años es el que mayor confianza manifiesta $(17,2 \%)$, presentando una recuperación de la confianza mayor que la población general y que el resto de los grupos etarios (30-49 y 50 años o más), siendo que en todas las ondas anteriores era el grupo que presentaba menores porcentajes de confianza. World Values Survey: ondas 1995, 1999, 2006 y 2013 de la variable «Confianza: partidos políticos» para Argentina. Base de datos disponible en http://www.worldvaluessurvey.org (consultado por última vez el 05/02/2018).
} 
por las organizaciones partidarias, especialmente por aquellas ligadas al espacio político de la ex presidenta Cristina Fernández de Kirchner (2007-2015).

Como veremos en la sección siguiente, estos fenómenos han atraído el interés de los investigadores, dando lugar a un nuevo y fértil campo de estudios. Sin embargo, la escasa atención prestada en las últimas décadas al estudio de las militancias partidarias en general, y a las organizaciones juveniles partidarias en particular, presenta el desafío de desarrollar herramientas conceptuales que permitan un estudio sistemático de estas formas de militancia juvenil.

Inspirado en una investigación doctoral sobre organizaciones juveniles partidarias en la Argentina reciente, el presente trabajo pretende realizar un aporte conceptual para el estudio de este tipo de organizaciones y militancias partidarias, desde un abordaje que busca dar cuenta de su construcción sociocultural.

En un primer apartado, revisaré la producción académica sobre militancias juveniles partidarias en América Latina, prestando especial atención a los estudios sobre Argentina en la última década. En un segundo apartado, propondré algunas herramientas para el estudio, desde la sociología política, de las organizaciones partidarias y sus militancias. En un tercer apartado, presentaré algunas propuestas conceptuales para el estudio de las organizaciones y militancias específicamente juveniles dentro de los partidos, desde un punto de vista cultural, organizacional y generacional.

\section{REVITALIZACIÓN DE LAS ORGANIZACIONES JUVENILES PARTIDARIAS EN ARGENTINA Y EMERGENCIA DE UN CAMPO DE ESTUDIOS}

Aunque el retorno de la democracia en Argentina, a fines de 1983, es considerado habitualmente como el inicio de un período de revitalización de la participación política en general y de la militancia juvenil en particular, y de que es reconocido el rol protagónico de las juventudes oficialistas durante el gobierno de Raúl Alfonsín (19831989), pocos son los estudios académicos de aquel período que se han ocupado de investigar estas u otras organizaciones partidarias juveniles durante los ochenta.

Estudios pioneros sobre participación política juvenil, como los de Braslavsky (1986) y Berguier et al. (1986), destacan la relevancia adquirida con el retorno democrático, de la participación en los partidos políticos, los sindicatos, los centros de estudiantes y las movilizaciones. Altamirano (1987), por esos años, estudia el discurso de la Junta Coordinadora Nacional, un espacio de dirigentes juveniles alfonsinistas. Sin embargo, parte del interés por la participación partidaria durante los años de Alfonsín se ha despertado más recientemente, quizás como reflejo del reverdecer de la militancia juvenil oficialista durante los gobiernos kirchneristas y del debate público que esta ha despertado. Así, algunos trabajos (Fernández, 2010; Muiño, 2011; Rodríguez, 2013) se han volcado a revisitar la militancia en la Junta Coordinadora Nacional, mientras otros se han interesado por la agrupación universitaria del radicalismo Franja Morada (Bultynch, 2008; Beltrán, 2013) o por la agrupación universitaria de centro-derecha UPAU (Arriondo, 2015). Más recientemente, Vázquez et al. (2017) han estudiado distintas formas de militancia juvenil en los ochenta, trazando comparaciones y paralelismos con la participación política juvenil contemporánea. 
Pasada la primavera del retorno democrático, el final de la década de los ochenta inaugura el debate acerca de la «apatía juvenil», que marcará una de las principales líneas de indagación de la década siguiente (Balardini, 2005). En contraste con la militancia revolucionaria de los sesenta y setenta, o con la efervescencia de la transición democrática, la relación de los jóvenes con la política fue caracterizada por muchos estudios de la década de los noventa en términos de apatía o desencanto (Kozel, 1996; Sidicaro y Tenti Fanfani, 1998; Urresti, 2000), dando cuenta de un distanciamiento de las generaciones más jóvenes respecto a las instituciones políticas.

Mientras algunos diagnósticos se centraron en el distanciamiento respecto a las formas de participación tradicionales, otros estudios posteriores, especialmente en el escenario abierto luego de la crisis política y socioeconómica de 2001, desplazaron su mirada hacia la participación por fuera del ámbito institucional o, en algunos casos, en rechazo a la política partidaria. De este modo, algunas investigaciones fijaron su atención en la participación juvenil en organizaciones de Derechos Humanos (Bonaldi, 2006), en el movimiento estudiantil (Bonavena et al., 2007, Núñez, 2008; Vommaro y Picotto, 2010), en los emergentes movimientos de desocupados (Vommaro y Vázquez, 2008; Vázquez, 2009) y en el ámbito de las organizaciones no gubernamentales (Beltrán y Malagamba Otegui, 2010). Otras, en cambio, se ocuparon de estudiar la politicidad de ciertas prácticas culturales juveniles (Semán y Vila, 1999; Svampa, 2001; Chaves, 2007; Citro, 2008).

En el marco de una relativa recomposición del sistema político y de recuperación de las capacidades estatales a partir de la llegada al gobierno de Néstor Kirchner (2003-2007), y especialmente a partir de la década siguiente, comienzan a publicarse estudios que destacan un proceso de repolitización (Natalucci, 2014) y, más concretamente, una «vuelta» de las generaciones jóvenes a la militancia dentro de ámbitos que parecían haber sido abandonados en la década de los noventa (Borobia et al., 2013; Natanson, 2013; Vommaro y Larrondo, 2013), como los sindicatos (Natalucci y Galimberti, 2015; Wolanski, 2015) y las organizaciones partidarias.

Si las postrimerías de la década del noventa y los años posteriores a la crisis de 2001 se caracterizaron por una territorialización de la militancia o una politización de lo territorial, con la emergencia de movimientos de desocupados y organizaciones «piqueteras» (Svampa y Pereyra, 2003; Delamata, 2004; Merklen, 2005), a partir del gobierno de Néstor Kirchner, muchas de estas organizaciones ingresaron al juego político partidario, participando en la implementación de políticas sociales en el territorio e integrándose dentro del espacio oficialista. Una línea de investigación reciente se ha ocupado de estudiar este pasaje de la militancia territorial y autónoma, no siempre identificada como juvenil, hacia la militancia partidaria o desde el Estado (Perelmiter, 2012; Natalucci, 2012; Pagliarone, 2012; Schuttenberg, 2012; Cura 2014).

La emergencia de la interpelación a la «juventud» en el discurso de los dirigentes partidarios es una de las notas distintivas del período. Vázquez (2013) estudia la génesis y la consagración de la juventud como «causa militante» en el kirchnerismo, mientras Flax (2013) estudia, desde la perspectiva del análisis del discurso, los modos en los que la juventud es enunciada en la retórica presidencial de Cristina Kirchner. Asimismo, la militancia juvenil ha despertado un fuerte debate en la esfera pública, especialmente a partir de la expansión y visibilización de la organización kirchnerista La Cámpora, luego de 2010. En consecuencia, otro grupo de trabajo se ha ocupado de 
estudiar el modo en el que la militancia juvenil kirchnerista ha sido representada por el periodismo (Cozachcow, 2015; Rocca Rivarola, 2014; Flax, 2014).

No obstante el intenso debate y visibilidad pública de las organizaciones partidarias juveniles durante los últimos años, la producción académica sobre este objeto se concentra principalmente en las juventudes kirchneristas, con trabajos como los de Vázquez y Vommaro (2012), Vázquez (2014), Garrido (2012) y Mutuverría (2017). Otras investigaciones se han ocupado de la militancia juvenil en el partido de Mauricio Macri, Propuesta Republicana (Grandinetti, 2014, 2015a, 2015b; Vommaro, Morresi y Belloti, 2015). Recientemente, también, se han realizado algunos estudios comparativos entre casos argentinos (Rocca Rivarola y Bonazzi, 2017; Vázquez, Rocca Rivarola y Cozachcow, 2018) y de estos con militancias partidarias de otros países (Rocca Rivarola, 2015; Diaz-Parra, Roca y Romano, 2016; Palacios-Valladares, 2016).

Mientras que la literatura reciente sobre política y juventudes en Argentina ha enfatizado la revalorización de las organizaciones partidarias en tanto espacios de participación, los trabajos acerca de otros países de América Latina coinciden, en cambio, en mostrar la distancia de las generaciones jóvenes respecto de las formas tradicionales de involucramiento, dando cuenta de una creciente desconfianza hacia a las instituciones políticas, que se refleja en una participación más baja en los procesos electorales y los partidos. Sin embargo, la literatura coincide en señalar que este distanciamiento de la política tradicional no implica una despolitización (Coe y Vandegrift, 2015; P. Vommaro, 2015). Al contrario, los jóvenes han ocupado un lugar protagónico en diversos procesos de movilización y acción colectiva de la última década en Chile (Ponce Lara, 2010), México (Meneses Rocha et al., 2017) y Brasil (Duarte, 2016), por mencionar los casos más relevantes. Así, han emergido nuevas formas de involucrarse en los asuntos públicos, nuevas prácticas y modos de organización (Correa y Manríquez, 2009; Seixas Silva y Rabello de Castro, 2014), que se caracterizan por la centralidad adquirida por las acciones directas y culturales, el uso de los medios digitales, la organización en redes informales y horizontales, la autonomía respecto a las organizaciones políticas y al Estado, y la aparición de una nueva agenda que no solo incluye reivindicaciones estudiantiles, sino también cuestiones de género, desigualdad social y violencia institucional.

Sin embargo, existe también un conjunto de estudios que se han interesado por la participación juvenil en organizaciones partidarias en la región durante la última década. Espinoza y Madrid (2010) publicaron un libro sobre las militancias juveniles en Chile, en el que analizan las formas de participación, la entrada a la militancia, la socialización política y las relaciones generacionales en siete organizaciones juveniles partidarias. También para el caso chileno, Arce Riffo (2013) analiza la participación de jóvenes militantes en la democracia interna de seis partidos. Cuna Pérez (2006) se ocupa de la relación de los jóvenes mexicanos con los partidos políticos, analizando tanto las visiones de los dirigentes juveniles partidarios como de los jóvenes «desencantados» con estos espacios de participación. En Perú, Ayala Abril (2017) estudia las motivaciones y los incentivos para la militancia entre los jóvenes del partido Solidaridad Nacional. Con el objetivo de relativizar el diagnóstico de la apatía juvenil y la falta de relevancia de los partidos políticos, Acosta (2011) estudia las prácticas de participación en las organizaciones juveniles partidarias de Colombia. También desafiando las miradas sobre la decadencia de la participación juvenil en los partidos, Castro Rocha (2009) toma el caso de los jóvenes del PT en Brasil y se ocupa de analizar las formas que asumen su compromiso político y sus carreras militantes. Lizbona 
Cohen (2012), finalmente, se vale de una perspectiva de género para estudiar la militancia juvenil en cuatro partidos uruguayos, preguntándose por las expectativas que los y las jóvenes militantes desarrollan en torno a sus carreras políticas.

\section{EL ESTUDIO DE LAS ORGANIZACIONES Y LAS MILITANCIAS PARTIDARIAS A TRAVÉS DE SUS ÁMBITOS DE SOCIABILIDAD Y RECLUTAMIENTO}

Los estudios sobre partidos políticos han señalado, desde hace ya varias décadas, una serie de transformaciones que han afectado tanto sus anclajes sociales como sus formas organizativas. Las estructuras sociales más complejas y fragmentadas, resultantes de los procesos de modernización, dieron lugar a formatos partidarios orientados a electorados múltiples y heterogéneos y, por lo tanto, menos anclados social e ideológicamente (Kirchheimer, 1980). Los partidos se habrían convertido, así, en emprendimientos electorales en manos de políticos profesionales y expertos (Panebianco, 1988), con una caída en el número y relevancia de sus miembros o militantes (Mair y Van Biezen, 2001; Dalton y Wattenberg, 2002; Scarrow y Gezgor, 2010; Whiteley, 2011; Van Haute y Gauja, 2015). De este modo, los partidos ya no serían principalmente mediadores entre el Estado y la sociedad, canalizando demandas de grupos sociales a través de políticas públicas, sino que tenderían a aparecer como brokers del Estado ante la sociedad (Katz y Mair, 1995, 2002, 2009) y a depender, cada vez más, de los recursos estatales para su supervivencia (Van Biezen y Kopecky, 2007).

Sin embargo, que los objetivos organizacionales de los partidos se encuentren más o menos autonomizados socialmente respecto a etapas previas, no significa que los partidos no tengan vínculos con instituciones y grupos sociales, ni que los miembros que los producen y reproducen no sean agentes posicionados en el espacio social, dotados de recursos materiales y simbólicos resultantes de sus prácticas en diversos ámbitos de sociabilidad. Las luchas intrapartidarias, la formación de facciones internas, los recursos movilizados para la conquista de posiciones de poder y los repertorios de acción de las militancias partidarias no pueden ser adecuadamente analizados si no se estudian relacionalmente las propiedades sociales de los agentes que hacen a la organización.

Es en este sentido que sugiero estudiar a las organizaciones partidarias como el producto de las interacciones de sus miembros en sus distintos niveles (afiliados, militantes, dirigentes adultos y juveniles, funcionarios, etc.) y de esas organizaciones con otras organizaciones y grupos sociales. Así, si consideramos que las organizaciones partidarias son entramados de relaciones sociales (Lagroye, 1994; Offerlé, 2004), no es aceptable que su entorno sea reducido únicamente a una serie de dimensiones políticoinstitucionales como el sistema electoral, el sistema de partidos o el régimen político. En consecuencia, argumento aquí que ese entorno restringido debe ser expandido más allá, para dar cuenta también de los medios sociales en los cuales las organizaciones partidarias nacen y se desarrollan, y sus militantes son reclutados y socializados (Sawicki, 1997, 2001, 2011).

Desde el punto de vista de los agentes, es decir, de los miembros de la organización en sus distintos niveles, este medio social se compone de circuitos de ámbitos de sociabilidad, esto es, de espacios de interacción perdurables, en los cuales los agentes desarrollan una socialización política continua (Bargel, 2009; Fillieule y Pudal, 2010; 
Fillieule, 2012, 2013). El enfoque que propongo busca estudiar a la militancia juvenil en su carácter procesual (Sawicki y Siméant, 2009), atendiendo a las trayectorias de los militantes, sus procesos de politización y socialización política. Dentro del estudio del medio social partidario, resulta significativo el análisis de lo que Gabriel Vommaro (2015) llama mundos sociales de pertenencia. El estudio de los mundos sociales de pertenencia que integran el medio social partidario permite dar cuenta de las formas de sociabilidad y prácticas a través de las cuales los militantes adquieren esquemas interpretativos y expresivos, saberes prácticos y repertorios de acción. Desde el punto de vista de la organización, el estudio de su medio social permite dar cuenta de los ámbitos de reclutamiento de sus militantes, cuadros dirigentes y funcionarios, y, de este modo, de la implantación del partido entre diferentes grupos sociales. Por otra parte, nos muestra las relaciones, no necesariamente formalizadas, que los miembros del partido establecen con otros ámbitos de sociabilidad y organizaciones vinculadas o no a la actividad política. En este sentido, permite estudiar al partido y a sus militantes más allá de sus fronteras formales, considerando en el análisis a las redes que no se encuentran formalmente integradas al partido, pero que conforman un recurso organizacional de este (Schwartz y Lowson, 2005). Esta es, posiblemente, una de las ventajas de este tipo de enfoque para el estudio de los partidos políticos y sus militancias en América Latina, dada la centralidad de las organizaciones informales en los partidos de la región (Levitsky, 2001; Freidenberg y Levitsky, 2007).

\section{LA «JUVENTUD» EN LAS ORGANIZACIONES PARTIDARIAS: ASPECTOS CULTURALES, ORGANIZACIONALES Y GENERACIONALES}

La elaboración de un enfoque conceptual para estudiar militantes y organizaciones partidarias que se autodefinen como juveniles me exime aquí de pretender dar solución a uno de los problemas teórico-metodológicos más recurrentes y complejos de las investigaciones sobre juventudes: el de la delimitación de los jóvenes como objeto. ${ }^{3}$ Sugiero prescindir, entonces, de las definiciones objetivistas de la juventud para estudiar, en cambio, los modos en los que la juventud es construida por los mismos agentes y sus organizaciones. Si, como argumenta Bourdieu (2001, 2000), la acción política tiene como objetivo hacer y deshacer grupos al producir las representaciones que los hacen visibles ante sí mismos y ante los demás, puede entenderse que la juventud, como principio de construcción de grupos y, por ello, de representación, sea materia de luchas en torno a su definición, que implican para sus portavoces la pretensión de actuar y hablar en nombre y a través de los «jóvenes». De este modo, la juventud resulta ser una categoría movilizada políticamente, dotada de atributos morales en disputa y utilizada para la conformación de organizaciones políticas que interpelan y construyen performativamente a ese grupo.

Desde una perspectiva relacional, los sentidos otorgados a la juventud pueden ser entendidos en sus usos hacia dentro y hacia fuera de la organización partidaria. Hacia adentro, sugiero analizarlos como un modo de estructurar y regular las interacciones de los agentes definidos como jóvenes y adultos en el partido. En este sentido, el foco del análisis estará en el lugar que ocupan los jóvenes dentro de la organización partidaria y las tareas que le son asignadas en tanto tales (Bargel 2009a, 2009b). Hacia afuera, por su parte, propongo estudiarlos a partir sus disputas con otros grupos políticos también

\footnotetext{
${ }^{3}$ Sobre este problema, puede consultarse Bourdieu (2008), Brunet y Pizzi (2013), Margulis y Urresti (1996), Mauger (1995, 2010) y Woodman y Bennett (2015).
} 
definidos como juveniles en torno a las propiedades legítimas de la juventud, y, por lo tanto, como un principio de legitimación política, así como de interpelación y producción de un colectivo al que se pretende representar políticamente. Así, el análisis empírico consistirá en objetivar la objetivación que los militantes y sus organizaciones hacen de la juventud, los modos en los que la hacen existir como categoría política, como grupo partidario interno y como electorado interpelado.

La formación de grupos etarios en una organización supone la delimitación de divisiones que, partiendo de la edad biológica, producen aquello que Bourdieu (2008) denomina edad social: la definición de aquella frontera de edad a partir de la cual pueden (o no) asumirse determinados roles y ocuparse determinadas posiciones de poder. Ser definido como joven o como adulto en un partido implica, necesariamente, una distribución del poder y de las prácticas entre aquellos que se encuentran en una etapa de minoridad y dependencia, y aquellos que han adquirido una membresía plena. Las organizaciones juveniles partidarias, en tanto ámbitos diferenciados de participación dentro del partido, traducen la idea de juventud como etapa de moratoria social o transición (Brunet y Pizzi, 2013; Mauger, 2010; Margulis y Urresti, 1996) en una moratoria política: una etapa de preparación y ejercitación necesaria para ingresar a la adultez partidaria y al ejercicio pleno de la actividad política en la organización.

Aun cuando las fronteras son negociables, los así definidos como adultos son quienes se encuentran dotados de mayores recursos organizacionales para definir esta división y regular el funcionamiento del partido y de su espacio juvenil. Esto, sin embargo, no implica la ausencia de disputas en relación con el lugar que ocupan los jóvenes dentro del partido o de la pertinencia misma de la división etaria, ni tampoco supone que los jóvenes no estén en condiciones de hacer de su juventud un atributo capitalizable para moverse ascendentemente en el partido. Para utilizar los términos de Panebianco (1988), las organizaciones juveniles brindan un incentivo selectivo a aquellos militantes interesados en hacer carrera, al facilitarles un espacio más accesible y con su propia estructura jerárquica interna para iniciar su ascenso dentro del partido. Por otra parte, para los líderes partidarios, crear sus propias agrupaciones o facciones juveniles les permite ganar visibilidad y prestigio dentro y fuera del partido. Contar con una militancia juvenil "propia», movilizable durante las campañas y los actos partidarios, es un recurso para legitimarse como dirigentes dentro del partido y para mostrarse cercanos a las generaciones jóvenes.

Las divisiones etarias y las formas en las que se organizan las relaciones entre grupos de edad dentro de los partidos pueden alcanzar diversos grados y formas de institucionalización. Algunos partidos poseen una única organización juvenil que busca agrupar y brindar un espacio de participación interno a todos los definidos como jóvenes, mientras otros no cuentan con una rama juvenil «orgánica» y permiten a sus dirigentes y líneas internas la creación de distintas agrupaciones juveniles. Al mismo tiempo, mientras algunos partidos fijan límites de edad que implican el abandono obligatorio de la organización juvenil, otros relegan la permanencia en estos espacios a la autoidentificación o a criterios informales. La institucionalización de umbrales de edad, al reforzar el carácter transicional de estos espacios y establecer un ritmo fijo de circulación para sus miembros, aparece como un obstáculo para la conformación de agrupaciones juveniles con liderazgos estables y relativamente autónomos respecto al partido que las contiene.

Al mismo tiempo que la juventud funciona como un principio para la división del trabajo y la distribución del poder dentro del partido, opera internamente también como un principio para la creación de organizaciones políticas y para la producción de una 
identidad basada en la alteridad etaria que, a diferencia de la adultez, se complementa y solapa con la identidad partidaria.

A su vez, las organizaciones juveniles son ámbitos de sociabilidad donde los militantes más jóvenes del partido encuentran pares generacionales con los que interactuar y establecer lazos. Así, según algunas investigaciones (Hooghe, Stolle y Stouthuysen, 2004; Bruter y Harrison, 2009), la existencia de organizaciones juveniles favorecería el reclutamiento y la retención de los ingresantes más jóvenes dentro de los partidos, brindando incentivos sociales e identitarios (Panebianco, 1988; Weber, 2017) específicos para este grupo.

Las organizaciones partidarias que se definen a sí mismas como juveniles —en algunos casos fijando un umbral de edad para sus miembros - tienen una composición relativamente homogénea en términos etarios $\mathrm{y}$, por lo tanto, sus miembros se han socializado y han ingresado al partido en un período de tiempo común. El carácter transicional o efímero de la participación en agrupaciones como estas —más aún cuando deben ser abandonadas al cumplir cierta edad- hace que los agentes que producen y reproducen a la organización juvenil se renueven más rápidamente que en el resto del partido, lo que la vuelve más permeable a los efectos de los procesos sociopolíticos recientes. Una de las consecuencias de esto - amplificada cuando existe una definición etaria institucionalizada de la juventud en el partido - es que los procesos de politización de estos militantes pueden ser estudiados en clave generacional y referidos a los contextos sociopolíticos en los que tuvieron lugar. En este sentido, el concepto de generación nos remite a la experiencia social de los acontecimientos históricos y los modos en los que estos operan como factores de politización, es decir, como instancias en las que se configuran ciertas formas de relación con el mundo político, se transforman o cristalizan sentidos respecto a la política, y se despierta o se pierde interés por la participación.

Resulta relevante aquí retomar la distinción conceptual realizada por Karl Mannheim (1993) entre posición generacional y conexión generacional. Mientras la primera refiere a una mera contemporaneidad de fechas de nacimiento, la conexión generacional da cuenta de la probabilidad de que, dada una posición generacional común, se participe de los mismos sucesos y experiencias sociales, y más específicamente, se lo haga desde una misma estratificación de la conciencia, esto es, que estas experiencias, en función de las trayectorias de vida de los agentes, ocupen una misma posición relativa en la configuración de sus modos de pensar y de vivir. Es el modo en el que esos sucesos son experimentados — en función de una trayectoria no solo vital, sino también social, que implica diversos ámbitos y formas de socialización - lo que provoca distintos posicionamientos y formas de intervención respecto a esa problemática generacionalmente compartida. Así, dentro de una misma conexión generacional pueden formarse diversas unidades generacionales orientadas hacia las mismas problemáticas, pero en sentidos que pueden ser incluso antagónicos. Es en torno a una unidad generacional que pueden organizarse grupos concretos, como los movimientos u organizaciones juveniles.

En este sentido, las categorías de Mannheim pueden servirnos para el estudio de las organizaciones partidarias juveniles, pudiendo dar cuenta tanto de la conexión entre militantes de distintos espacios políticos, resultante de la participación en cierta problemática generacionalmente compartida y vinculada a determinados procesos sociopolíticos comunes, como de los diversos posicionamientos, antagonismos y fronteras a partir de los cuales estos agentes, aún conectados generacionalmente, se diferencian en términos políticos. 
Por otra parte, en el estudio de las organizaciones partidarias juveniles, lo generacional aparece no solo como un recurso analítico del que nos valemos para dar cuenta de ciertos procesos de politización relativamente homogéneos, en función de la participación en determinadas formas de experiencia social e histórica comunes (Fillieule, 2013, 2012; Bargel 2009c; Ihl, 2002; Sirinelli, 1989), sino también como un aspecto tematizado por los mismos militantes a partir de la producción de narrativas generacionales (Aboim y Vasconcelos, 2014). La noción de narrativa (Richardson, 1990; Kohler Riessman, 2012) implica considerar las explicaciones de los agentes sobre sus procesos de politización como racionalizaciones (Giddens, 1984), construidas retrospectivamente a partir de su experiencia actual de militancia. En estas narrativas, los agentes producen sentido desde sus posiciones actuales acerca de sus producciones de sentido pretéritas y organizan desde el presente su experiencia del pasado (Bourdieu, 2011).

En consecuencia, para reconstruir los procesos de politización en clave generacional, podemos valernos de la construcción que los mismos militantes hacen de aquellos acontecimientos que marcaron su interés por la política y su acercamiento al partido. Así, pensar una organización juvenil desde su dimensión generacional implica reconstruir los sentidos que adquieren para sus miembros, retrospectivamente, estos procesos sociopolíticos en su propia politización, en búsqueda tanto de una conexión generacional —en su relación con otras juventudes partidarias-, como de la unidad generacional de sus posicionamientos. El enfoque que he presentado permite, por lo tanto, vincular el punto de vista de los agentes acerca de su propia politización con los procesos sociopolíticos en los que esta tiene lugar.

\section{Palabras finales}

Con el propósito de fortalecer un campo temático que ha ganado vigencia en la Argentina reciente y, al mismo tiempo, de fundamentar la pertinencia de un abordaje desde la sociología política, he esbozado en las secciones anteriores una serie de herramientas para construir este objeto desde múltiples dimensiones.

En primer lugar, propuse un estudio de las organizaciones partidarias y su militancia juvenil a través de los ámbitos de sociabilidad y reclutamiento que componen su medio social. En segundo lugar, presenté una serie de consideraciones para estudiar el carácter «juvenil» en estas organizaciones: los sentidos partidarios atribuidos a la categoría, sus usos organizacionales y la dimensión generacional de los procesos de movilización e involucramiento político de los militantes.

He sugerido, asimismo, que investigar estas organizaciones juveniles se presenta como una vía de acceso para el estudio de las organizaciones partidarias en general, permitiéndonos dar cuenta de: i) uno de los modos en los que se organiza el trabajo político y se distribuye el poder interno; ii) uno de los principales canales para el reclutamiento (y retención) de militantes; iii) uno de los espacios internos desde el cual se desarrollan carreras políticas ascendentes y se adquieren recursos para la actividad política; iv) un ámbito de militancia de base por excelencia que permite estudiar los medios sociales y la sociabilidad partidaria; v) un principio de legitimación e interpelación política utilizado por las organizaciones partidarias y sus dirigentes, y vi) los procesos de politización o despolitización (y partidización o despartidización de la participación política) en clave generacional. 
Si, como lo demuestra la evidencia empírica, los partidos políticos siguen siendo canales relevantes para la selección de dirigentes y la toma de decisiones públicas, la participación de las generaciones jóvenes en ellos es crítica para el presente y el futuro de nuestras democracias. Así, la comprensión de los modos de involucramiento político de los jóvenes y de las condiciones en las que estos son interpelados y reclutados por los partidos resulta importante no solo para el estudio de la política o de las juventudes, sino también para el fortalecimiento de la democracia.

RECIBIDO: 20 DE NOVIEMBRE DE 2018

ACEPTADO: 27 DE MARZO DE 2019

\section{REFERENCIAS BIBLIOGRÁFICAS}

AвOIM, S. y VASCONCELOS, P. (2014). From political to social generations: A critical reappraisal of Mannheim's classical approach. European Journal of Social Theory, 7(2), 165-183.

Acosta, G. (2011). Jóvenes en la política partidaria. Una aproximación a las organizaciones de juventud vinculadas a los partidos políticos en Colombia. Anagramas, 10(19), 51-68.

AltAmiRAnO, C. (1987). La Coordinadora. Elementos para una interpretación. En J. C. PORTANTIERO y J. Nun (Eds.), Ensayos sobre la transición democrática en la Argentina. Buenos Aires: Puntosur.

ArCe Riffo, J. (2013). Dilemas de la democracia interna en los partidos políticos de Chile. ¿Cómo participan los jóvenes militantes? Andamios, 7, 86-102.

ARRIONDO, L. (2015). De la UCeDe al PRO. Un recorrido por la trayectoria de los militantes de centro derecha de la Ciudad de Buenos Aires. En G. Vommaro y S. MORRESI (Eds.), «Hagamos equipo». PRO y la construcción de la nueva derecha en Argentina. Los Polvorines: UNGS Ediciones.

Ayala Abril, H. (2017). Ser militante en el Perú: Aproximaciones desde la juventud de Solidaridad Nacional. Revista Argumentos, 1(11), 31-38.

BAEza Correa, J. y SANDOval ManRíquez, M. (2009). Nuevas prácticas políticas en jóvenes de Chile: Conocimientos acumulados 2000-2008. Revista Latinoamericana de Ciencias Sociales, Niñez y Juventud, 7(2), 1379-1403.

BALARDinI, S. (2005). ¿Qué hay de nuevo, viejo? Una mirada sobre los cambios en la participación política juvenil. Nueva Sociedad, 200, 96-107.

BARGEL, L. (2009a). «La jeunesse qui bouge a changé de camp!» Des usages partisans de la catégorie «jeunesse». Mouvements, 59, 83-89.

(2009b). Les organisations de jeunesse des partis politiques. Agora Débat/jeunesses, 52, 75-88.

(2009c). Socialisation politique. En O. FILLIEUle, et al. (Eds.), Dictionnaire des mouvements sociaux. París: Presses de Science Po. 
Beltrán, G. y MALAGAMBA Otegui, R. (Eds.) (2010). Jóvenes y ONG'S: una agenda pendiente. Buenos Aires: Aurelia Rivera.

Beltrán, M. (2013). La Franja. De la experiencia universitaria al desafío del poder. Buenos Aires: Aguilar.

Berguier, R., HeCKer, E. y SCHIFrin, A. (1986). Estudiantes secundarios: sociedad y política. Buenos Aires: Centro Editor de América Latina.

BonALDI, P. (2006). Hijos de desaparecidos. Entre la construcción de la política y la construcción de la memoria. En E. JELIN y D. SEMPOL (Eds.), El pasado en el futuro: los movimientos juveniles. Buenos Aires: Siglo XXI.

Bonavena, P., Califa, J. y Millán, M. (Eds.) (2007). El movimiento estudiantil argentino. Historia con presente. Buenos Aires: Ediciones Cooperativas.

Borobia, R., KROPfF, L. y NÚÑEZ, P. (2013). La participación política juvenil post2001/3. En R. Borobia, L. KROPFF, y P. NúÑEz (Eds.), Juventud y participación política. Más allá de la sorpresa. Buenos Aires: Noveduc.

Bourdieu, P. (2000). La delegación y el fetichismo político. En Cosas dichas. Madrid: Gedisa.

(2001). La representación política. En El campo político. La Paz: Plural.

(2008). La «juventud» es sólo una palabra. En Cuestiones de Sociología. Madrid: Akal.

(2011). La ilusión biográfica. Acta Sociológica, 56, 121-128.

Braslavsky, C. (1986). La juventud argentina: informe de situación. Buenos Aires: Centro Editor de América Latina.

BRunET, I. y Pizzi, A. (2013). El enfoque nominalista de la juventud. Una alternativa crítica a la perspectiva funcionalista. Revista Latinoamericana de Ciencias Sociales, Niñez y Juventud, 1(11), 51-62.

BRUTER, M. y HARRISOn, S. (2009). Tomorrow's Leaders? Understanding the Involvement of Young Party Members Six European Democracies. Comparative Political Studies, 42(10), 1259-1291.

Bultynch, D. (2008). La Franja Morada de los 80. Ponencia presentada en I Jornadas de Historia de la Universidad Argentina, La Plata, Argentina.

Castro Rocha, D. (2009). Jeunes du Parti des travailleurs et crise du militantisme. Agora débats/jeunesses, 2(52), 89-104.

Chaves, M. (2007). Los espacios urbanos de jóvenes en La Plata. Avá. Revista de Antropología, 213-215.

CITRO, S. (2009). El rock como ritual adolescente. Trasgresión y realismo grotesco en los recitales de Bersuit. TRANS. Revista transcultural de música, 12. 
Coe, A. y VAndegrift, D. (2015). Youth Politics and Culture in Contemporary Latin America: A Review. Latin American Politics and Society, 57(2), 132-153. DOI:10.1111/j.1548-2456.2015.00271.x

CozAchCOW, A. (2015). Juventudes y política: usos de la militancia juvenil en La Cámpora en medios nacionales durante la campaña electoral 2013. Questión, 1(47), 7594.

CunA PÉREZ, E. (2006). Reflexiones sobre el desencanto democrático. El caso de los partidos políticos y los jóvenes en la ciudad de México. Sociológica, 21(61), 95-133.

CurA, M. (2014). De militar los barrios a militar el Estado. Etnografía sobre modalidades de acción política, formación de militancias y compromiso político juvenil en Argentina. Antípoda, 20, 49-71.

Dalton, R. y Wattenberg, M. (Eds.) (2002). Parties without partisans: Political change in advance industrial democracies. Oxford: Oxford Press.

DelamatA, G. (2004). Los barrios desbordados. Las organizaciones de desocupados del Gran Buenos Aires. Buenos Aires: Eudeba-Libros del Rojas.

Diaz-PARRA, I., RocA, B. y Romano, S. (2015). Political activists' frames in times of post-politics: evidence from Kirchnerism in Argentina and Podemos in Spain. Contemporary Social Science, 10(4), 386-400. DOI: 10.1080/21582041.2016.1218042

DuARTE, A. (2016). Juventude, movimentos sociais e participação política no Brasil entre os anos de 2013 a 2015. Revista Educativa, 19(1), 884-901. DOI: http://dx.doi.org/10.18224/educ.v19i3.5442

ESPINOZA, V. y MADRID, S. (2010). Trayectoria y eficacia política de los militantes en juventudes políticas. Estudio de la élite política emergente. Santiago: USACH-PNUD.

FERNÁNDEZ, J. C. (2010). La Junta Coordinadora Nacional: innovaciones discursivas y organizativas en el radicalismo (1968/1983). Ponencia presentada en V Jornadas de Historia Política, Mar del Plata, Argentina.

FILlieule, O. (2012). Travail, familie, politisation. En I. SAINSAUlieu y M. SuRdEZ (Eds.), Sens politiques du travail. París: Armand Colin Recherches.

(2013). Political socialization and social movements. En D. SNOw, et al. (Eds.), The Wiley-Blackwell Encyclopedia of Social and Political Movements. Oxford: Wiley.

Fillieule, O. y Pudal, B. (2010). Sociologie du militantisme. Problématisations et déplacement des méthodes d'enquête. En É. Agrikoliansky, I. Sommier y O. FILLIEUle (Eds.), Penser les mouvements sociaux. Conflits sociaux et contestations dans les societies contemporaines. París: La Découverte Recherches.

FLAX, R. (2013). La representación de los jóvenes en la retórica presidencial de Cristina Fernández de Kirchner. En M. Vitale y P. SAlazAr (Eds.), Rhetoric South America. Cape Town: Africa Rhetoric Publishing.

(2014). La representación de los militantes peronistas en la prensa argentina: la cobertura del diario La Nación del acto organizado por la juventud peronista el 11 de marzo de 2011. Logos: Revista de Lingüística, Filosofía y Literatura, 24(1), 15-29. 
FREIDENBERG, F. y LEVITSKY, S. (2007). Organización informal de los partidos en América Latina. Desarrollo Económico, 46(184), 539-568.

GARRIDO, N. (2012). Cibermilitancia 2.0 La juventud kirchnerista en la Argentina de hoy. Sociedad y Equidad, 4, 91-108.

GIDDENS, A. (1984). The Constitution of Society. Outline of a Theory of Structuration. Los Ángeles: University of California Press.

GrandinetTI, J. (2014). «Jóvenes de espíritu». Usos y sentidos de la juventud en el PRO. Sociales en Debate, 6, 29-38.

(2015a). «Mirar para adelante». Tres dimensiones de la juventud en la militancia de Jóvenes PRO. En G. VOMMARo y S. Morresi (Eds.), «Hagamos equipo». PRO y la construcción de la nueva derecha en Argentina. Los Polvorines: Ediciones UNGS.

(2015b). De misionar a militar. La participación en voluntariados solidarios católicos como forma de socialización política entre los militantes de Jóvenes PRO. En P. DALle, et al. (Eds.), Prácticas del oficio. Buenos Aires: CLACSO.

Hooghe, M., Stolle, D. y Stouthuysen, P. (2004). Head start politics. The Recruitment Function of Youth Organizations of Political Parties Belgium (Flanders). Party Politics, 10(2), 193-212.

IHL, O. (2002). Socialisation et événements politiques. Revue française de science politique, 52(2/3), 125-144.

KATZ, R. y MAIR, P. (1995). Changing Models of Party Organization and Party Democracy: The Emergence of the Cartel Party. Party Politics, 1(1), 5-28.

(2002). The Ascendancy of the Party Public Office: Party Organizational Change Twentieth-Century Democracies. En R. GunTher, et al. (Eds.), Political parties: old concepts and new challenges. Oxford: Oxford University Press. 766.

(2009). The Cartel Party thesis: a restatement. Perspectives on Politics, 7(4), 753-

KIRCHHEIMER, O. (1980). El camino hacia el partido de todo el mundo. En K. LENK y F. Neumann (Eds.), Teoría y sociología críticas de los partidos políticos. Barcelona: Anagrama.

KoHLER RiEsSMAn, K. (2012). Analysis of personal narratives. En J. GubriUm (Ed.), The SAGE Handbook of Interview Research. Los Ángeles: SAGE.

Kozel, A. (1996). Los jóvenes y la política. Modulaciones de un escepticismo general. En M. MARgulis (Ed.), La juventud es más que una palabra. Buenos Aires: Biblos.

LAgroye, J. (1994). Sociología política. México: Fondo de Cultura Económica.

LEVITSKY, S. (2001). An Organized Disorganization: Informal Organization and the Persistence of Local Party Structures in Argentine Peronism. Journal of Latin American Studies, 33(1), 29-66. 
Lizbona Cohen, A. (2012). La militancia de las y los jóvenes en Uruguay: ¿su carrera política? Mirada Joven, 2, 7-24.

Mair, P. y VAN BIEzen, I. (2001). Party Membership in Twenty European Democracies, 1980-2000. Party Politics, 7(1), 5-21.

Mannheim, K. (1993). El problema de las generaciones. Revista Española de Investigaciones Sociológicas, 93(62), 193-242.

MARgulis, M. y URRESTI, M. La juventud es más que una palabra. En M. MARGULIS (Ed.), La juventud es más que una palabra. Ensayos sobre cultura y juventud. Buenos Aires: Biblos.

MAuger, G. (1995). Jeunesse: l’âge des classements. Essai de définition sociologique d'un âge de la vie. Recherches et prévisions, 40, 19-36.

(2010). Jeunesse: essai de construction d’objet. Agora débats/jeunesses, 56, 9-24.

Meneses Rocha, M., Ortega Gutiérrez, E. y Urbina Cortés, G. (2017). Youth and Political Participation. \#Yosoy132 and the struggle for Freedom of Expression in Mexico. Communication y Society, 30(1), 87-103.

Merklen, D. (2005). Pobres ciudadanos. Las clases populares en la era democrática. Argentina, 1983-2003. Buenos Aires: Gorla.

Muiño, O. (2011). Los días de la Coordinadora. Políticas, ideas, medios y sociedad: 1968-1983. Buenos Aires: Corregidor/Ediciones IML.

Mutuverría, M. (2017). Militantes y Estado. Prácticas de Oficio, 18(2), 1-13.

NATALUCCI, A. (2012). Los movimentistas. Expectativas y desafíos del Movimiento Evita en el espacio kirchnerista (2003-2010). En A. NATALUCCI y G. PÉREZ (Eds.), Vamos las bandas. Organizaciones y militancia kirchnerista. Buenos Aires: Nueva Trilce.

(2014). La cultura política en el kirchnerismo: dos hipótesis sobre la politización. Sudamérica, 3, 155-171.

NAtalucci, A. y Galimberti, C. (2015). Juventud(es) sindical(es): identidades políticas y lógicas de acción (AMBA, 2009-2015). Socio Debate. Revista de Ciencias Sociales, 1(2), 98-130.

NATANSON, J. (2013). El retorno de la juventud. Movimientos de repolitización juvenil en nuevos contextos urbanos. Nueva Sociedad, 243, 92-103.

NúÑEZ, P. (2008). La redefinición del vínculo juventud-política en Argentina: un estudio a partir de las representaciones y prácticas políticas juveniles en la escuela secundaria y media. Revista Latinoamericana de Ciencias Sociales, Niñez y Juventud, 6(1), 149-190.

OfFERLÉ, M. (2004). Los partidos políticos. Santiago: LOM Ediciones. 
Pagliarone, M. F. (2012). Piqueteros y funcionarios. Transformaciones de la FTV en el kirchnerismo. En A. NATAluCCI y G. PÉREZ (Eds.), Vamos las bandas. Organizaciones y militancia kirchnerista. Buenos Aires: Nueva Trilce.

Palacios-Valladares, I. (2016). With or without them. Contemporary student movements and parties in the southern cone. The Latin Americanist, 60(2), 243-268, DOI: $10.1111 /$ tla.12076

Panebianco, A. (1988). Political parties: organization and power. Cambridge: Cambridge University Press.

Perelmiter, L. (2012). Fronteras inestables y eficaces. El ingreso de organizaciones de desocupados a la burocracia asistencial del Estado. Argentina (2003-2008). Estudios Sociológicos, 30(89), 431-456.

Ponce LARA, C. (2010). Juventud, movilizaciones e identidad política en Chile. Ponencia presentada en VI Congreso CEISAL, Toulouse, Francia.

RICHARDSON, L. (1990). Narrative and Sociology. Journal of Contemporary Ethnography, 19(1), 116-135.

Rocca Rivarola, D. (2014). El libro «bueno» y el libro «malo» sobre La Cámpora: sus aportes para la investigación social sobre la militancia oficialista. Estudios, 31, 259267.

(2015). Vínculos y formas de la militancia oficialista. Un modo de adaptación a las condiciones de fluctuación política en Argentina y Brasil. Papeles de Trabajo, 9(15), 170-198.

Rocca Rivarola, D. y Bonazzi, M. (2017). El «otro» militante. Concepciones y prácticas militantes al interior del kirchnerismo y el macrismo. POSTData Revista de reflexión y análisis político, 22(2), 655-686.

RodRíGueZ, G. (2013). ¿Heredarás el viento? Liderazgos fundacionales y cambio generacional en el proceso político argentino a treinta años de 1983. Observatorio Latinoamericano, 12, 276-290.

SAWICKI, F. (1997). Les réseaux du Parti Socialiste: sociologie d'un milieu partisan. París: Belin.

(2001). Les parties politiques comme entreprises culturelles. En D. CEFAÏ (Ed.), Les cultures politiques. París: PUF.

(2011). Para una sociología de los entornos y las redes partidistas. Revista de Sociología, 25, 37-53.

SCARrow, S. y GEzGOR, B. (2010). Declining memberships, changing members? European political party members in a new era. Party Politics, 16(6), 823-843.

Schuttenberg, M. (2012). La trayectoria política de Libres del Sur 2003-2011. Reconfiguración identitaria, alianza y ruptura con el kirchnerismo. En A. NATALUCCI y G. PÉREZ (Eds.), Vamos las bandas. Organizaciones y militancia kirchnerista. Buenos Aires: Nueva Trilce. 
SCHWARTZ, M. y LOWSON, K. (2005). Political parties: social bases, organization, and environment. En JANOSKI, T. et al. (Eds.), The handbook of political sociology: states, civil societies, and globalization. Nueva York: Cambridge.

Seixas Silva, C. y Rabello de Castro, L. (2014). Brazilian Youth Activism: In Search of New Meanings for Political Engagement? Alternatives, 39(3), 187-201. https://doi.org/10.1177\%2F0304375415569389

SEMÁn, P. y VilA, P. (1999). Rock chabón e identidad juvenil en la Argentina neoliberal. En D. FILMUS (Ed.), Los 90. Política, sociedad y cultura en América Latina y Argentina en fin de siglo. Buenos Aires: FLACSO-Eudeba.

SidicARO, R. y TENTI FAnfani, E. (Comps.) (1998). La Argentina de los jóvenes. Entre la indiferencia y la indignación. Buenos Aires: UNICEF-Losada.

SIRINELLI, J. (1989). Génération et histoire politique. Vingtième Siècle. Revue d'histoire, 22, 67-80.

Svampa, M. (2001). Identidades astilladas. De la patria metalúrgica al heavy metal. En M. SVAMPA (Ed.), Desde abajo. La transformación de las identidades sociales. Buenos Aires: Biblos.

Svampa, M. y Pereyra, S. (2003). Entre la ruta y el barrio. La experiencia de las organizaciones piqueteras. Buenos Aires: Biblos.

URresti, M. (2000). Paradigmas de participación juvenil: un balance histórico. En S. BALARDINI (Ed.), La participación social y política de los jóvenes en el horizonte del nuevo siglo. Buenos Aires: CLACSO.

Van Biezen, I. y Kopecky, P. (2007). The State and the Parties: Public Funding, Public Regulation and Rent-Seeking Contemporary Democracies. Party Politics, 13(2), 235254.

Van Haute, E. y Gauja, A. (2015). Introduction: Party membership and activism. En E. Van Haute y A. Gauja (Eds.), Party Members and Activists. Abingdon: Routledge.

VÁzQuez, M. (2009). La política desde abajo: narrativas militantes de jóvenes desocupados y desocupadas en Argentina. Revista Latinoamericana de Ciencias Sociales, Niñez y Juventud, 7(1), 423-455.

VÁZQUEZ, M. (2013). En torno a la construcción de la juventud como causa pública durante el kirchnerismo: principios de adhesión, participación y reconocimiento. Revista Argentina de Estudios de Juventud, 1(7), 1-28.

(2014). «Militar la gestión»: una aproximación a las relaciones entre activismo y trabajo en el Estado a partir de las gestiones de gobierno de Cristina Fernández de Kirchner en Argentina. Apuntes, 41(74), 71-102.

VÁzquez, M. y CozachCow, A. (2017). Activismo juvenil en partidos con gestiones de gobierno a nivel subnacional en Argentina (2007-2015). Revista de Sociologia e Política, 64(25), 47-72. 
VÁzQUez, M. y Vommaro, P. (2012). La fuerza de los jóvenes: aproximaciones a la militancia kirchnerista desde La Cámpora. En A. NATAluCCI y G. PÉREz (Eds.), Vamos las bandas. Organizaciones y militancia kirchnerista. Buenos Aires: Nueva Trilce.

VÁzquez, M., Rocca Rivarola, D. y Cozachcow, A. (2018). Compromisos militantes en juventudes político-partidarias (Argentina, 2013-2015). Revista Mexicana de Sociología, 80(3), 519-548.

VÁzquez, M., Vommaro, P., NúñEz, P. y Blanco, R. (Eds.) (2017). Militancias juveniles en la Argentina democrática. Trayectorias, espacios y figuras de activismo. Buenos Aires: Imago Mundi.

Vommaro, G. (2015). Contribución a una sociología política de los partidos. Los mundos sociales de pertenencia y las generaciones políticas de PRO. En G. VOMMARO y S. MORRESI (Eds.), «Hagamos equipo». PRO y la construcción de la nueva derecha en Argentina. Los Polvorines: UNGS Ediciones.

Vommaro, G., Morresi, S. y Bellotti, A. (2015). Mundo PRO. Anatomía de un partido fabricado para ganar. Buenos Aires: Planeta.

Vommaro, P. (2015). Juventudes y políticas en la Argentina y en América Latina. Buenos Aires: Grupo Editor Latinoamericano.

Vommaro, P. y LARRONDO, M. (2013). Juventudes y participación política en los últimos treinta años de democracia en la Argentina: conflictos, cambios y persistencias. Observatorio Latinoamericano, 12.

Vommaro, P. y PicotTo, D. (2010). Jóvenes y política: las agrupaciones estudiantiles independientes de la Universidad de Buenos Aires. Nómadas, 32.

Vommaro, P. y VÁzquez, M. (2008). La participación juvenil en los movimientos sociales autónomos de la Argentina. El caso de los Movimientos de Trabajadores Desocupados (MTDs). Revista Latinoamericana de Ciencias Sociales, Niñez y Juventud, 6(2), 485-522.

WeBer, R. (2017). Political participation of young people in political parties. A literature review of influencing factors on the macro, meso and micro level of participation. Z Politikwiss, 27, 379-396.

Whiteley, P. (2011). Is the party over? The decline of party activism and membership across the democratic world. Party Politics, 17(1), 21-44.

WOLANSKI, S. (2015). Organizar la Juventud. Un estudio etnográfico comparativo sobre experiencias juveniles de organización gremial. En Jóvenes en movimientos. Buenos Aires: CLACSO.

Woodman, D. y BennetT, A. (Eds.) (2015). Youth cultures, transitions, and generations. Bridging the gap in youth research. Londres: Palgrave. 